cause hospitalization than those treated with olanzapine and quetiapine, lower risk of BPD-I-related hospitalization than quetiapine and risperidone, and fewer hospital days than quetiapine in a Medicaid population.

Funding. Sunovion Pharmaceuticals Inc.

\title{
Discovering Non-Invasive Biomarkers Predictive of Opioid Use Disorder Treatment Response
}

Amir Levine, $\mathrm{MD}^{1}$, Kelly Clemenza, $\mathrm{MS}^{1}$,
Shira Weiss, $\mathrm{HBSc}^{1}, \mathrm{Adam}^{1}$ Bisaga, $\mathrm{MD}^{1}$, Erez Eitan, $\mathrm{PhD}^{2}$,
Maria Carla Gerra, $\mathrm{PhD}^{3}$, Claudia Donnini, $\mathrm{PhD}^{3}$,
Gilberto Gerra, $\mathrm{MD}^{4}$ and Benjamin Garcia, $\mathrm{PhD}^{5}$

${ }^{1}$ Columbia University, New York, NY, USA, ${ }^{2}$ NeuroDex Incorporated, Natick, MA, USA, ${ }^{3}$ University of Parma, Parma, Emilia-Romagna, Italy, ${ }^{4}$ United Nations Office on Drugs and Crime, Vienna, Austria, and ${ }^{5}$ University of Pennsylvania, Philadelphia, PA, USA

\section{Presenting Author: Kelly Clemenza}

liquid chromatography-mass spectrometry. EV miRNA cargo was determined by RNA sequencing.

Results. We found one lipid class and six miRNAs that differed significantly between the naltrexone group and the methadone and control groups. We also found that histone H3acK9acK14 was increasingly acetylated in PMBCs from both the methadone and naltrexone groups compared to controls.

Discussion. Naltrexone, which is used in treatment of OUD and other substance use disorders as well as disorders of impulse control, was found to have multiple potential corresponding molecular signatures that can be identified after long-term treatment. It remains to be seen if these markers can also be a good predictor for treatment response. In addition, significant gender differences in EV content are found between men and women with OUD, which supports the importance of examining changes in response to treatment in a gender informed way.

\section{Could Improved Microcirculation Reverse the Effects of Fetal Alcohol Syndrome? A Review}

\author{
Arman Phillipe Yavari, BA
}

University of Colorado at Boulder, Boulder, CO, USA

Presenting Author: Arman Phillipe Yavari

\begin{abstract}
Fetal alcohol spectrum disorders, (FASDs) are a spectrum of neurodevelopmental and cognitive conditions, caused by the consumption of alcohol in varying amounts in utero that affect an estimated $1-5 \%$ of the general population in the United States. The effects of the spectrum of disorders are lifelong, resulting in often significant learning difficulties, cognitive deficits, and behavioral issues. It is known that blood flow and microcirculation to the brain are affected by the consumption of alcohol in utero, and that microcirculation and neurology are intimately linked, with changes in the former having drastic effects on the latter. The purpose of the current review is to analyze whether improvement in microcirculation in the infant or child with FASDs could affect quality of clinical outcomes in the spectrum of disorders, or lead to the improvement of disorderrelated symptoms. Scientific evidence from the literature suggests that, in theory, improving cerebral microcirculation in the infant or young child with FASDs could lead to a corresponding improvement in neurological health, and potentially an improvement in cerebral development, which may in theory lead to a lessening of symptoms. Further review into the connection between microcirculation and neurological health, and correspondingly, clinical outcomes in patients with neurological deficits, is warranted.
\end{abstract}

